\title{
GENRES DE TEXTES EN CIRCULATION DANS LA SPHËRE DU TRAVAIL D’ENSEIGNEMENT ET DANS LA FORMATION PROFESSIONNELLE DE L'ENSEIGNANT
}

\author{
TEXT GENRES THAT CIRCULATE IN THE SPHERE OF TEACHING WORK AND IN \\ TEACHER PROFESSIONAL DEVELOPMENT
}

\author{
Eliane Gouvêa Lousada \\ Universidade de São Paulo, São Paulo, São Paulo, Brasil \\ elianelousada@uol.com.br
}

Resumé : Dans cet article, nous avons pour but de discuter des relations entre langage et travail, en focalisant sur le rôle potentiellement formatif des genres textuels dans le contexte du travail d'enseignement. Nous présenterons les genres qui circulent dans les situations de travail enseignant et ceux qui peuvent être produits à des buts formatifs, en distinguant ceux qui circulent dans la sphère de travail ceux qui ont un potentiel d'apprentissage et/ou de développement. Notre étude se base sur l'orientation épistémologique de l'Interactionnisme Social et se sert de trois cadres théoriques : celui de l'Interactionnisme Socio-discursif, d'une part; et ceux de la Clinique de l'Activité et de l'Ergonomie de l'Activité des Professionnels de l'Education, d'autre part. Nous montrerons, de manière spécifique, une analyse d'une autoconfrontation et nous discuterons de son potentiel pour le développement des enseignants et pour la formation. Nous discuterons ainsi du rôle des verbalisations sur la situation de travail dans le processus de formation de l'enseignant, à travers des genres produits en situation de travail qui constituent une activité symbolique dans laquelle le langage devient un instrument de formation et de développement.

Mots-clés : Travail enseignant ; Langage ; Développement ; Auto-confrontation

Abstract: In this article, we aim to discuss the relationships between language and work, focusing on the potentially formative role of text genres in the context of teaching work. We will present the genres which circulate in teaching work situations and those which can be produced for developmental purposes, by distinguishing those which circulate in the sphere of work those which have a potential of learning and / or development. Our study is based on the epistemological orientation of Social Interactionism and uses three theoretical frameworks: that of Socio-discursive Interactionism, on the one hand; and those of the Activity Clinic and Ergonomics of the Activity of Education Professionals, on the other hand. We will show, in a specific way, an analysis of a self-confrontation interview and we will discuss its potential for the development of teachers and for training. We will thus discuss the role of verbalizations on the work situation in the teacher development process, through genres produced in work situations which constitute a symbolic activity in which language becomes an instrument of education and development.

Key-words: Teaching work; Language; Development; Self-confrontation interview

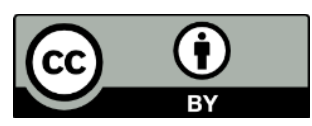




\section{INTRODUCTION}

Si les préoccupations à propos des effets du travail sur la santé sont anciennes et remontent à la Renaissance, voire à l'Antiquité, les études sur les rapports homme-travail ont débuté dans le XXe siècle, entre les deux guerres (Laville, 2001). Les études sur le rôle du langage et sur les rapports langage et travail, à leur tour, datent de la fin du XXe siècle. En reprenant l'histoire, on observe que c'était d'abord sur le travail de production que les préoccupations des chercheurs se sont concentrées. Cependant, c'était à partir de 1986 que certains chercheurs, notamment le réseau Langage et Travail (Boutet, 2001, 2016), ont commencé à s'intéresser aux rapports entre langage et travail et à ce qu'ils appelaient « le travail symbolique », c'est-à-dire le travail qui n'a pas pour but la modification de la matière première en produit. C'était dans la continuité de ces réflexions que Boutet $(2001,2016)$ a commencé à parler de la part langagière du travail, un concept encore plus large que celui de travail symbolique, car il permet de s'intéresser à la part du travail qui est réalisée à travers ou à partir du langage. Pour elle, le langage est une partie indissociable du travail, quoique, dans certains moments socio-historiques, comme à l'époque du taylorisme, on ait fait plusieurs efforts pour l'éliminer.

Selon Boutet (2016), la part langagière du travail se voit, à l'oral ou à l'écrit, de manière variable, dans les pratiques langagières dans le but soit de réaliser les activités de travail, soit de les commenter, soit encore d'échapper aux tâches du travail. De notre part, nous soutenons que cette part langagière du travail s'organise en genres de textes (Bronckart, 1999), qui circulent dans les situations de travail, les organisent, leur donnent une forme et permettent leur réalisation. Cette part langagière est différente d'un travail à l'autre et, pour certains métiers, elle est prépondérante, comme c'est le cas du travail enseignant et de tant d'autres comme le droit, la pshychologie, etc. (Kostulski, 2015).

Lorsqu'on parle de la part langagière du travail, selon Boutet $(2001,2016)$ on n'a pas affaire à des corpus autonomes, déconnectés des conditions matérielles de leur énonciation : leur contexte de production est essentiel pour leur compréhension. Voilà pourquoi il faut prendre en compte ce contexte lors de l'analyse et l'interprétation des données produites dans différentes situations de travail. Pour cette auteure (Boutet, 2001, 2016), l'étude de la part langagière du travail prend en compte plusieurs travaux dont ceux de M. Bakthine et L. Vygotski, qui voient le langage non pas comme une faculté naturelle, mais comme un processus historique sous la dépendance des conditions matérielles et sociales.

Dans cet article, nous visons à discuter des relations entre langage et travail, en focalisant sur le rôle potentiellement formatif des genres textuels dans le contexte du travail d'enseignement. De manière plus spécifique, nous présenterons les genres qui circulent dans les situations de travail enseignant et ceux qui peuvent être produits à des buts formatifs, en distinguant ceux qui circulent dans la sphère de travail ceux qui ont un potentiel d'apprentissage et/ou de développement. Nous montrerons ainsi le rôle des verbalisations sur la situation de travail dans le processus de formation de l'enseignant, à travers des genres produits en situation de travail qui constituent une activité symbolique dans laquelle le langage devient un instrument de formation et de développement.

\section{LENTILLES POUR COMPRENDRE ET ANALYSER LES GENRES TEXTES PRODUITS EN SITUATION DE TRAVAIL}

Pour comprendre et analyser les genres textuels produits en situation de travail enseignant, nous adoptons comme orientation épistémologique générale une perspective 
historico-culturelle, c'est-à-dire, l'Interactionnisme Social tel que proposé par Vygotski (1934/1997). Parmi les plusieurs études réalisées par Vygotski (1934/1997), les notions qui nous intéressent et dont nous nous servons dans cet article sont celles d'instrument et de formation de concepts. Pour cet auteur, les rapports entre l'homme et le milieu sont médiatisés au travers d'instruments, qui peuvent être matériels (dans les cas des outils les plus variés pour modifier, transformer le milieu), ou symboliques, comme tous les systèmes de signes, dont le langage. A travers ces instruments, l'homme modifie la nature et agit sur le monde et sur les autres.

Dans les recherches conduites par Vygotski (1934/1997), le langage a un rôle primordial, car il permet à l'homme, non seulement de communiquer en agissant sur les autres, mais aussi d'agir sur lui-même, en contrôlant ses propres processus psychiques. Le langage permet de représenter, nommer, catégoriser les objets du monde, autrement dit, formuler des « concepts ». Selon lui, la généralisation, la capacité à formuler des concepts est une caractéristique intrinsèque de l'être humain. Friedrich (2010, p. 103), dans ses études sur la pensée vygotskienne, affirme que : «En d'autres termes : utiliser un concept ne signifie pas simplement généraliser, mettre ensemble les choses dans le monde, mais penser le monde, ce qui peut conduire à des liens entre les choses dans le monde qui ne sont pas des liens factuels ». Elle ajoute encore que la généralisation est possible à travers le mot, voilà pourquoi le rôle du langage est essentiel, en tant qu'instrument psychologique, dans la construction du psychisme humain. Selon Friedrich (2010, p. 102) :

\begin{abstract}
Le mot ne clôt pas la généralisation, mais porte la généralisation ; il n'est pas son expression mais le support du processus même. Dans l'approche de Vygotski, le mot n'est ni le résultat de la généralisation ni sa condition immuable mais le « lieu » où la pensée prend naissance (Friedrich, 2010, p. 102).
\end{abstract}

Ainsi, pour Vygotski, «le mot permet de penser le monde et pas seulement de le représenter. » (Friedrich, 2010, p. 102).

Comme nous le verrons par la suite, les notions que nous venons de présenter sont essentielles pour les études que nous réalisons, car elles permettent de montrer comment les textes, appartenant à différents genres produits dans leurs situations de travail, permettent aux enseignants de «penser leur travail ».

Aussi essentielles que les études vygotskiennes soient pour comprendre les rapports entre pensée et langage, cet auteur n'a pas développé, pour plusieurs raisons, y compris son décès prématuré, une théorie du langage qui permette d'analyser les textes produits, dans notre cas, dans les situations de travail enseignant. Nous avons ainsi adopté un courant, basé sur l'Interactionnisme Social, qui a justement pour but d'étudier le rôle du langage, du fonctionnement discursif, dans le développement humain. Il s'agit de l'Interactionnisme Sociodiscursif (ISD) proposé par Bronckart $(1999,2008)$ qui étudie notamment l'agir langagier et propose, outre des travaux sur la Didactique des Langues ${ }^{1}$ qu'il n'est pas question d'aborder ici, des apports pour analyser les productions langagières présentes dans les différentes activités humaines, dont le travail.

Le modèle de l'ISD est descendant et propose que l'on parte d'un ensemble d'hypothèses sur le contexte de production de textes, pour ensuite passer à l'analyse des éléments discursifs et linguistiques. Ces hypothèses concernent le contexte physique et le

\footnotetext{
${ }^{1}$ Le modèle d'analyse textuel a été proposé par l'ISD (Bronckart, 1999) justement dans le but de contribuer à un renouvellement de l'enseignement des langues. Il n'en reste pas moins que ce modèle est assez complet et a servi, depuis deux décennies, à analyser des textes produits en situation de travail (Bulea, 2010).
} 
contexte socio-subjectif qui sont à l'origine de la production textuelle. On peut les résumer comme suit :

Tableau 1 - Contextes physique et socio-subjectif

\begin{tabular}{cc}
\hline Contexte physique & Contexte socio-subjectif \\
\hline Emetteur & Enonciateur (rôle social de l'émetteur) \\
Récepteur & Destinataire (rôle social du récepteur) \\
Lieu de production & Lieu social \\
Moment de production & Objectifs
\end{tabular}

Source : Bronckart, 1999.

A partir d'une compréhension du contexte de production, l'analyse des textes selon le modèle de l'ISD s'organise à partir de trois niveaux qui se chevauchent, mais qu'il convient de séparer à des fins d'analyse. L'infra-structure générale est le premier niveau d'analyse et elle se décline en : plan global des contenus thématiques, types de discours et éventuelles séquences. Le plan global des contenus thématiques peut être compris comme un résumé du texte. Let type de discours est une catégorie proposée par Bronckart (1999) à partir des études proposées par Benveniste, Weinrich et Simonin-Grumbach en essayant de mettre en rapport, respectivement, les distinctions entre discours et histoire et entre monde commenté et monde narré. Il en résulte une distinction entre le monde de l'exposer (conjoint), qui se partage entre impliqué (discours interactif) et autonome (discours théorique) et le monde du relater, partagé également en impliqué (récit interactif) et autonome (narration) (Bronckart, 1999). Les types discursifs représentent des modes d'énonciation, des attitudes du producteur lors de la production langagière. Pour l'identification des types de discours il est également utile de se servir des mécanismes de cohésion verbale, raison pour laquelle Bronckart (2019) a placé cette catégorie, initialement analysée dans le deuxième niveau d'analyse, ici. Enfin, selon Bronckart (1999) les séquences textuelles peuvent être présentes ou non dans les textes. Il s'inspire d'Adam pour les proposer et suggère six types de séquence : descriptive, narrative, argumentative, injonctive, explicative et dialogale, sans compter le degré zéro de la séquence narrative (script) et le degré zéro des séquences argumentative et explicative (schématisation).

Le deuxième niveau d'analyse est celui des mécanismes de textualisation, qui assurent la cohérence thématique au texte. Il se compose des opérations de cohésion nominale (reprises anaphoriques de plusieurs formes) et de connexion, qui sont responsables de la macroorganisation du texte. Pour analyser la cohésion nominale dans les textes oraux, nous avons fait appel à des théories compatibles, mais qui focalisent la cohésion nominale dans des textes oraux. Ainsi, nous avons utilisé les études de Koch (2002/2013), Mondada (2002) et Sitri (1996, 2006), qui proposent d'envisager l'analyse des sens construits de manière évolutive tout au long des interactions orales. En fait, en reconnaissant un statut différencié au texte oral par rapport au texte écrit dans le processus de progression référentielle, ces auteures proposent qu'on intègre plusieurs types de cohésion qui prennent en compte des phénomènes sémantiques, référentiels et thématiques. Ainsi, nous avons pu identifier non seulement les éléments nominaux et pronominaux qui assuraient la cohésion des extraits analysés, mais nous avons essayé de retrouver les séries cohésives complexes, organisées autour des mêmes " idées », qu'elles soient représentées par des noms, des pronoms, des adjectifs, des verbes ou même des adverbes. En effet, lorsque Koch (2002/2013) propose des catégories pour analyser la progression référentielle, elle indique que, dans les formes nominales référentielles, les choix lexicaux ont un rôle important dans l'argumentation.

Koch (2002/2013) affirme que la continuité référentielle n'implique pas des référents toujours stables et le texte n'est pas construit selon une continuité progressive linéaire. Dans les 
analyses que nous présentons dans cet article, nous observerons comment une idée autour de la quantité de vocabulaire enseignée dans un cours de base de français comme langue étrangère se construit à travers une reprise nominale qui se réalise à travers un emploi métaphorique d'un terme emprunté à un autre discours.

Mondada (2002) a proposé à plusieurs reprises l'idée que, tout au long de l'interaction, des objets de discours se construisent et se transforment, au sein d'une dimension interdiscursive qui prend en compte l'énonciateur et le destinataire. Sitri $(1996,2006)$ part de cette idée pour affirmer qu'au cours de l'interaction les objets de discours acquièrent de nouveaux sens, ils se modifient. Dans nos recherches, cela nous a permis de comprendre comment, dans les interactions pendant lesquelles les professeurs parlent de leur travail, de nouveaux sens sur les actions du métier sont construits et se modifient.

Finalement, nous avons le troisième niveau proposé par Bronckart (1999, 2019), les mécanismes de prise en charge énonciative, responsables de la cohérence interactive du texte, qui représentent le troisième niveau d'analyse textuelle. A ce niveau-là, les catégories d'analyse sont la gestion des voix qui sont présentes, de manière plus ou moins explicite, dans le texte, et les modalisations. Bronckart (1999) a proposé trois types de voix : de l'auteur empirique, des personnages et voix sociales. Quant aux modalisations, en se basant sur d'autres auteurs qui les ont étudiées, Bronckart (1999) propose : les modalisations logiques (union des modalisations épistémiques et aléthiques) et qui ont affaire à des phénomènes perçus comme possibles, probables, certains, etc. ; déontiques, de l'ordre de la permission, du devoir, de l'obligation ; appréciatives, qui se basent sur des jugements émis par l'énonciateur par rapport au contenu thématique ; et, finalement, pragmatiques, proposées par Bronckart (1999) pour indiquer la perception de l'énonciateur par rapport aux capacités d'action des acteurs.

Une fois le cadre théorique et méthodologique qui sert de base à nos analyses expliqué, passons à la présentation des présupposés théoriques et méthodologiques qui nous permettent de comprendre et d'interpréter les données du point de vue du travail enseignant.

\section{PERSPECTIVE POUR ANALYSER LA PART LANGAGIÈRE DU TRAVAIL: TRANSFORMER LES SITUATIONS DE TRAVAIL POUR LES COMPRENDRE}

En partant des concepts vygotskiens (Vygotski, 1934/1997) comme ceux de développement, instrument et relations entre la pensée et le langage, la Clinique de l'Activité combine deux traditions différentes : l'ergonomie francophone et la psychopathologie du travail (Clot, 2001a), dont la proposition est de comprendre le travail pour le transformer. Justement, la Clinique d'Activité inverse ce binôme, en proposant de transformer le travail pour le comprendre. Cette idée part de la conception vygotskienne (Vygotski, 1934/1997) selon laquelle il est nécessaire de mettre un corps en mouvement pour étudier son développement, en créant ainsi des conditions qui permettent aux travailleurs de modifier leur propre situation de travail.

Pour atteindre cet objectif, des chercheurs comme Clot (2001) ont repris certaines méthodes, comme l'Instruction au Sosie proposée par Ivar Oddone dans les années 70. D'autres, comme Faïta et Vieira (2003), ont proposé la méthode de l'autoconfrontation. L'idée est de faire en sorte que ces méthodes soient des instruments pour que l'expérience du travailleur par rapport à son travail ne se limite pas à une expérience directe, mais puisse être observée à distance, c'est-à-dire, en faisant en sorte que les travailleurs soient des observateurs de leur propre activité professionnelle. La transformation du statut subjectif d'observation, c'est-à-dire, le fait que le travailleur, à travers l'enregistrement audio ou vidéo proposés par les méthodes, devient observateur de sa propre activité professionnelle, donne à ces méthodes le caractère 
«indirect», au sens vygotskien, car elles permettent au sujet d'accéder à son expérience à un autre moment, en se distanciant de l'expérience directe et en faisant en sorte que l'observation de l'activité professionnelle plus tard, à travers des enregistrements, devienne une autre expérience. Selon Clot (2001, p. 10), l'expérience vécue devient ainsi une façon de vivre d'autres expériences. Ce développement subjectif est, pour lui, un développement de la conscience. L'autoconfrontation devient ainsi un instrument psychologique (Clot, 2001, p. 9) pour que les travailleurs deviennent les protagonistes de l'observation et de l'analyse de leur travail et de celui de leurs pairs.

L'autoconfrontation (Faïta \& Vieira, 2003), la méthode utilisée dans notre dispositif de formation, se déroule selon les étapes suivantes :

- premièrement, observation des situations de travail, par l'intervenant et discussions avec les travailleurs sur leurs difficultés à exécuter le travail. A partir de ces discussions, les travailleurs choisissent les situations de travail qu'ils souhaiteraient filmer et ceux qui seront filmés dans l'exercice du métier. Il doit y avoir au moins deux travailleurs qui souhaitent être filmés tout en exerçant la même activité professionnelle. Ce long moment d'observation et de discussion, qui précède le tournage, est appelé « constitution du collectif»;

- ensuite, le filmage des situations de travail choisies par les travailleurs ;

- après le filmage, les travailleurs sont invités à regarder la vidéo de leur activité professionnelle, chacun à son tour avec l'intervenant. Celui-ci pose des questions sur « l'étrangeté » qu'il perçoit dans l'activité professionnelle du travailleur, pour que le travailleur voie son activité d'un autre point de vue, s'éloignant des actions qu'il effectue mécaniquement, sans se demander pourquoi. Il s'agit d'une autoconfrontation simple (ACS), qui se fait avec chacun des travailleurs filmés. Elle est également filmée

- à un moment postérieur, les deux travailleurs rencontrent l'intervenant, pour regarder les deux vidéos, un travailleur commentant l'activité de l'autre. L'intervenant règle le débat et pose également des questions. L'entretien se déroule sur la base de l'étrangeté, comme pour l'ACS, par rapport à la même activité, mais cette fois-ci réalisée par une autre. Ce moment, appelé autoconfrontation croisée (ACC), est également filmé ;

- enfin, ce travail réalisé avec seulement quelques travailleurs est restitué au collectif. Les travailleurs filmés, qui ont participé aux autoconfrontations, choisissent des extraits qu'ils souhaitent partager avec leurs collègues, en gardant à l'esprit les difficultés au travail évoquées lors des premières rencontres. La rencontre a lieu avec le collectif des travailleurs qui font l'observation collective des vidéos sélectionnées par les volontaires et il y a une discussion sur les aspects choisis par eux dans la première étape du travail. A ce moment-là, l'objectif est de provoquer des changements, des transformations dans la situation de travail, opérés par les travailleurs eux-mêmes.

Dans notre cas, nous avons utilisé la méthode de l'autoconfrontation dans un but formatif, mais toujours en ayant une visée développementale sur l'activité des enseignants de français comme langue étrangère, en visant à la transformation de la situation de travail. Malgré cette petite différence, nous avons gardé quelques points importants de l'AC, pour permettre d'avoir le même rôle dans le développement des enseignants: nous avons conservé, avec le groupe d'enseignants qui travaillent ensemble mais qui sont aussi en formation, un petit collectif et nous avons essayé d'écouter leurs difficultés, avant de commencer le filmage; le filmage des 
cours était une demande des professeurs et nous avons suivi toutes les étapes proposées par la méthode; à la fin, nous avons fait la restitution au « collectif» des enseignants. Notre objectif, dès le départ, était de développer l'activité de travail des enseignants en formation. De cette façon, nous nous éloignons de ceux qui n'utilisent la méthode de l'AC que pour collecter des données pour la recherche et nous conservons l'idée que le but premier de l'AC est celui de proposer la transformation des situations de travail, le développement de l'activité de travail (Clot et al., 2001) et l'élargissement du pouvoir d'agir des enseignants (Clot, 2008).

$\mathrm{Si}$, dans la Clinique de l'Activité, des interventions sont proposées dans diverses activités professionnelles, au sein du groupe de chercheurs de l'Ergonomie de l'Activité des Professionnels de l'Éducation (ERGAPE), l'accent est mis sur le travail d'enseignement, d'où notre intérêt à discuter de certains points qu'ils ont abordés.

Tout d'abord, il semble important de souligner que ces chercheurs (Faïta, 2004, Saujat, 2004) ont utilisé les méthodes indirectes pour proposer des dispositifs de formation des enseignants, comme nous l'avons fait. Sur la base de l'approche vygotskienne qui met l'accent sur le rôle de l'autre dans l'apprentissage et dans le développement, ces chercheurs ont fait des propositions de formation initiale ou continue des enseignants dans lesquelles le déplacement du contexte, "l'expérience vécue, comme mode de vivre de nouvelles expériences », peut agir comme moteur de développement, comme le soulignent Clot et al (2001). Sur la base du concept de ZPD (zone de développement proximal) de Vygotski (1997), ces auteurs ont proposé que, dans les ACC, en proposant un débat entre pairs qui rassemble des enseignants plus et moins expérimentés, différentes perceptions pour le même problème se posent, ce qui montre le rôle prédominant de l'autre dans l'apprentissage, comme moteur de développement. C'est, en effet, le déplacement du contexte acteur-enseignant, dans la situation de travail, vers l'observateur de son activité, qui permet une sémiotisation de l'expérience vécue, permettant de vivre une nouvelle expérience qui peut être à l'origine d'un développement possible.

Dans cette perspective, les travaux développés par l'équipe ERGAPE, élucident le rôle de l'AC dans la formation des enseignants et montrent que cette méthode peut contribuer à établir un pont entre les connaissances épistémiques, construites dans les réunions pédagogiques ou les sessions de formation (dans lesquelles nous abordons des textes théoriques et la planification matériel didactique) et les savoir-faire praxéologiques (liés à l'action, à la mise en œuvre de l'activité d'enseigner et à sa co-analyse ultérieure par le biais de l'AC). A la jonction de ces deux ordres de connaissance, nous sommes plus proches de ce que Bulea et Bronckart (2010) proposent pour la formation des enseignants, c'est-à-dire, l'union de deux ordres d'objectifs : ceux d'ordre épistémique et ceux d'ordre praxéologique.

\section{APPORTS MÉTHODOLOGIQUES POUR COMPRENDRE LES GENRES TEXTUELS EN CIRCULATION ET/OU PRODUITS EN SITUATION DE TRAVAIL}

Dans le but d'analyser la part langagière du travail, plusieurs classifications ont été proposées par des chercheurs. Nous ne pouvons pas les aborder toutes dans cet article, rappelons simplement deux parmi elles : celle de Nouroudine (2002) et celle de Bronckart (2004). La classification de Nouroudine (2002, p. 28), en partant d'autres auteurs, propose simplement une distinction entre deux « langages sur le travail » : celui des protagonistes du travail et celui des chercheurs sur le travail. Pour lui, aucun n'a une pertinence exclusive, tous les deux peuvent participer à un processus dialogique et dialectique dans lequel les deux se confronteront pour co-élaborer un langage sur le travail d'un nouveau genre. 
Bronckart (2004) a également proposé une classification de textes produits en situation de travail qui prend en compte les textes qui circulent dans les situations de travail et les textes qui peuvent être produits à des fins de recherche. Dans sa classification, l'auteur propose qu'on considère le travail situé dans l'entour-amont de l'agir, celui qui précède la réalisation des tâches et qui peut être considéré, grosso-modo comme le travail prescrit. Le travail réel (ou réalisé) découle du travail prescrit (sans être sa reproduction exacte, bien évidemment), c'està-dire, c'est la tâche exécutée. Autrement dit, ce sont les conduites des actants qui peuvent être retenues par des enregistrements audio ou vidéo. L'auteur mentionne également des textes qui peuvent être produits avant ou après l'exécution de la tâche : les textes d'entretiens avec les actants qui relèvent du travail interprété ou évalué, véhiculant, ainsi, un point de vue interne de la réalisation du travail. Enfin, il propose une dernière catégorie, celle des textes d'interprétation par les chercheurs, en tant qu'observateurs externes. Le schéma proposé par Bronckart (2004, p. 119) est le suivant :

Figure 1 : Textes en situation de travail

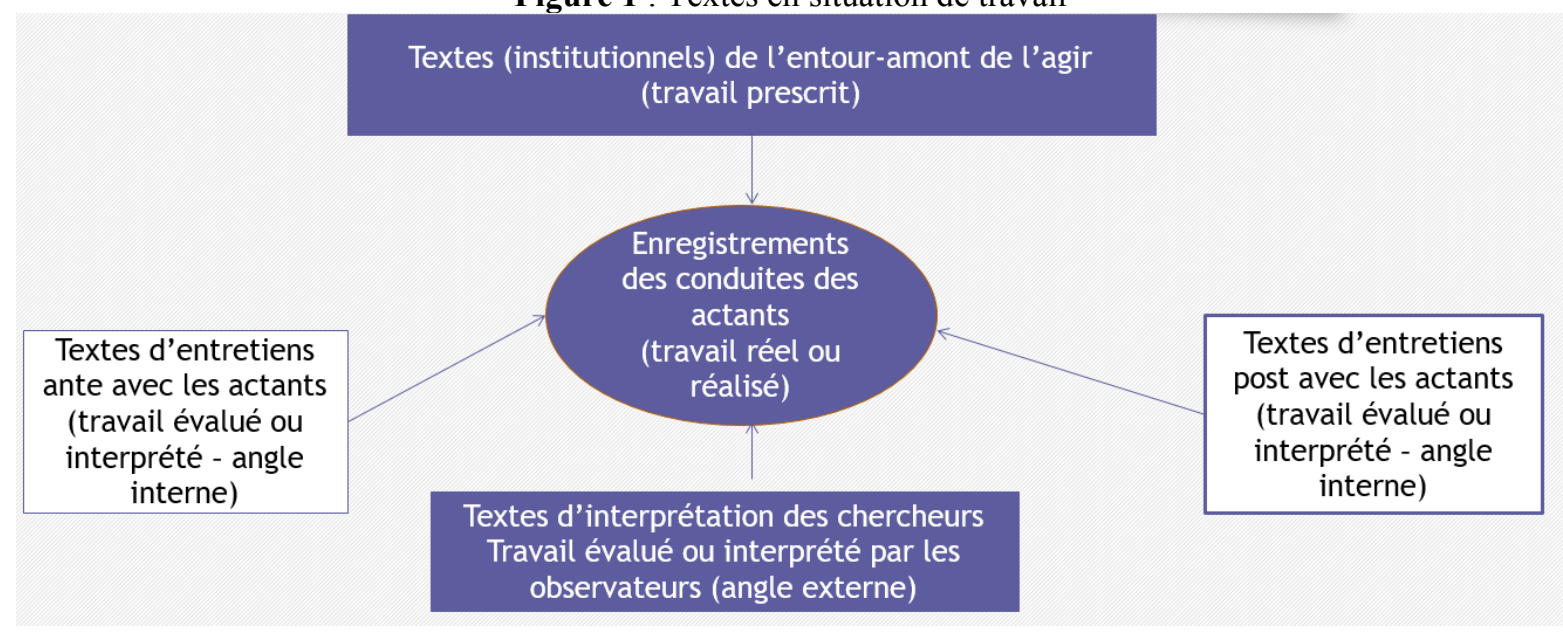

Source : Bronckart (2004, p. 119).

Nous nous sommes basées sur la classification proposée par Bronckart (2004), mais nous proposons de prendre également en compte les textes produits à des fins formatives, ce qui nous a menées à prendre en compte la proposition de Nouroudine (2002, p. 28). Selon cet auteur, il y aurait aux moins deux « langages sur le travail » : celle des protagonistes du travail, d'un côté, et celle des chercheurs, sans que l'une ne soit plus pertinente ni valide que l'autre. Pour lui, un langage sur le travail, approprié à son objet, passe par un processus dialogique et dialectique entre ces deux langages qui se confrontent (Nourodine, 2002, p. 28).

Nous avons ainsi proposé le schéma suivant, en nous basant sur le schéma de Bronckart (2004), mais en en prenant en compte les textes pour former : 
Figure 2 : Textes en situation de travail enseignant

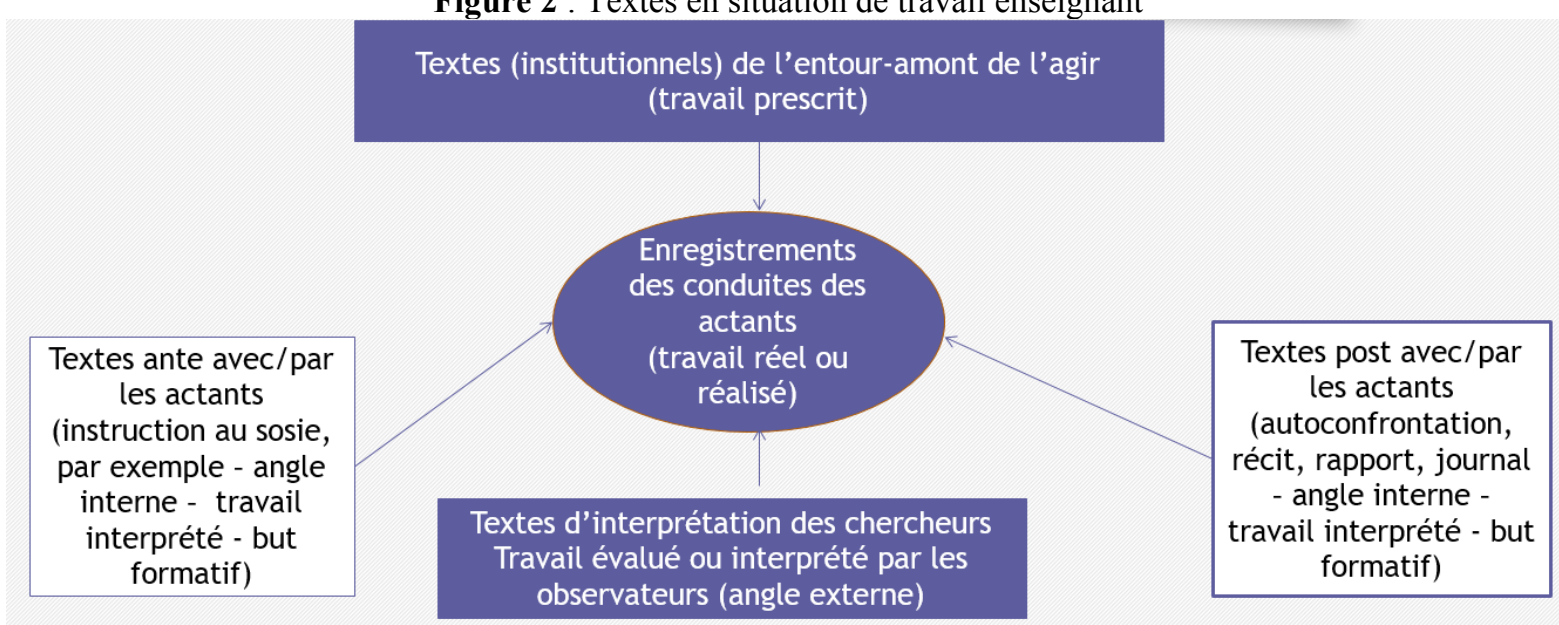

Source : adaptée de Bronckart (2004, p. 119).

A partir de ce schéma, nous avons également pris en compte les deux niveaux de réalisation du langage présents dans le travail de Nouroudine (2002) :

- Le premier niveau proposé par Nouroudine (2002) serait celui du langage produit par les protagonistes du travail, qui peut relever du travail prescrit (textes de l'entouramont de l'agir) ou du travail réalisé (enregistrements, photos, filmages des conduites des actants, mais aussi les textes produits par le protagoniste, pour la réalisation de son travail). A notre sens, le chercheur peut, bien évidemment, recueillir ces textes pour les étudier, cependant il ne s'agit pas de faire produire du langage exprès, pour l'intervention, la recherche ou la formation. Les genres de textes que nous trouvons à ce niveau sont extrêmement variés, car ils dépendent des activités sociales qui exigent l'usage du langage au travail, mais en voici quelques exemples : conversation dans diverses situations de travail (téléventes, client-vendeur, médecin-patient, etc.), ordonnances, plaidoiries, parmi tant d'autres.

- Le deuxième niveau proposé par Nouroudine (2002) serait celui du langage produit par les intervenants, les chercheurs ou les formateurs, c'est-à-dire, celui qui est produit à des fins de recherche ou de formation. A notre avis, lorsqu'on pense à un but de formation, d'apprentissage et/ou de développement, on peut penser que, dans ce deuxième cas, l'intervenant, le chercheur ou le formateur crée des situations où les protagonistes produisent des textes, verbalisent à propos de leur travail, dans le but de transformer le travail, l'étudier ou former par/pour le travail. Les genres de textes dans ce deuxième cas sont aussi variés, mais ils sont plus limités par les contraintes des situations d'intervention, recherche, formation, ce qui réduit leur nombre, puisqu'on cherche des activités susceptibles de créer des verbalisations sur le travail. En voici quelques exemples: rapport, récit d'expérience, journal, entretiens variés (d'instruction au sosie, d'autoconfrontation, d'explicitation, etc.).

Depuis plus d'une dizaine d'années (Lousada, 2006), nous étudions les textes de ce deuxième niveau, ce qui nous a menés à identifier une série de caractéristiques de certains parmi ces genres de textes, notamment: le récit, le rapport, l'instruction au sosie et l'autoconfrontation. Pour ce faire, nous nous sommes basés sur le modèle d'analyse textuel de Bronckart $(1999,2008,2019)$, mais aussi sur des apports d'autres auteurs tels que Maingueneau (2001), Authier-Revuz (2001), Mondada (2002) et Sitri (1996, 2006). C'est vers les 
caractéristiques énonciatives, discursives et linguistiques de l'autoconfrontation que se tourne la prochaine section.

\section{L'AUTOCONFRONTATION: COMBINER RÉCIT DE L'EXPÉRIENCE VÉCUE ET ACTIVITÉ COMMENTÉE POUR PERMETTRE DE PENSER LE TRAVAIL}

Pour atteindre les objectifs que nous poursuivons dans cet article, voyons les caractéristiques que nous avons identifiées dans les autoconfrontations, sur les plans énonciatif, discursif et linguistique, à partir du modèle proposé par Bronckart $(1999,2019)$ :

- deux plans énonciatifs : le cours filmé qui est reconstitué, en récit interactif et l'interaction en cours, en discours interactif;

- le recours à ces deux types discursifs met en parallèle, confronte, deux mondes : le monde narré et le monde commenté, ce qui permet la réflexion. Dans cette situation, lorsque l'enseignant verbalise à propos de son travail reconstitué par le moyen de la vidéo, il revit son expérience, pense sur son vécu, son ressenti, autrement dit, il pense le travail, pour reprendre le terme utilisé par Friedrich (2010) à propos de Vygotski (1997) ;

- présence de séquences dialogales, qui contiennent plusieurs pauses et hésitations, ce qui montre une pensée en cours de réalisation et qui nous montre encore que l'enseignant « pense le travail ».

- grande fréquence d'insertion de voix : de l'auteur empirique, de personnages (les apprenants, la coordination, les collègues, etc.), sociales (de la didactique, des documents officiels/institutionnels, du collectif de travail, etc.), selon ce que propose Bronckart (1999). Dans nos recherches, nous avons bien exploité la catégorie d'insertion de voix à partir de quelques catégories proposées par des auteurs comme Authier-Revuz (2001), Maingueneau (2001), et nous avons décelé plusieurs voix sociales et de personnages qui nous ont aidés à comprendre les influences de ces voix sur le travail enseignant (Lousada, 2006 ; Lousada, 2017). Plus récemment, nous avons identifié deux types de voix de l'auteur empirique : celle que l'enseignant reconstitue à partir du cours, dans les autoconfrontations (voix du langage intérieur reconstitué) et celle que l'enseignant utilise, pendant l'autoconfrontation, comme s'il pensait à voix haute (voix du langage intérieur extériorisé), pour «penser le travail »;

- présence de plusieurs modalisations de toutes sortes (appréciatives, logiques, déontiques et pragmatiques), qui montrent l'attitude des enseignants par rapport à des aspects de leur travail, les cours qu'ils ont donnés, leurs manières de faire. Ces modalisations montrent que l'enseignant réfléchit sur le travail et partage ses attitudes, ses manières de l'envisager, en discours interactif, pendant les autoconfrontations ;

- plus récemment, en prenant en compte la nature de nos données, surtout des textes oraux, nous nous sommes intéressés à des auteurs qui analysent la construction d'objets de discours au long des interactions verbales. Mondada (2002) étudie le développement et la transformation d'objets dans la durée du discours. Dans ces recherches et aussi dans celles de Sitri $(1996,2006)$, on observe les objets de discours dans leur dimension inter-discursive (Sitri, 2006 : 115) et on voit comment les objets de discours acquièrent de nouveaux sens, comment ils se modifient tout au long de l'interaction. 
A notre sens, le caractère développemental de l'autoconfrontation vient de ces caractéristiques: le fait de combiner des verbalisations sur l'expérience vécue (en récit interactif), mais en même temps de promouvoir un espace (en discours interactif) pour les commenter, en contribuant au débat interprétatif (Bronckart, 2008) ; le fait d'intégrer plusieurs voix qui contribuent aussi au débat interprétatif, car elles véhiculent des voix en accord, mais aussi en désaccord, avec ce que l'enseignant exprime ; la possibilité d'exprimer plusieurs points de vue, des interprétations, sur les contenus thématiques évoqués, au travers des modalisations ; et, finalement, la possibilité de co-construire des généralisations sur des aspects du travail, en puisant sur des termes issus des situations de travail, parfois des termes quotidiens, parfois des termes de la didactique, mais résignifiés car employés dans un autre contexte pour caractériser l'action de l'enseignant et pour en trouver ou restituer un (nouveau) sens dans sa situation particulière de travail.

Ces caractéristiques ont été observées dans plusieurs recherches, dans plusieurs contextes, mais, surtout, dans celui des cours d'extension de français de la Faculté de Lettres, dans lequel on forme des enseignants débutants ou peu expérimentés.

L'intervention dont l'extrait nous présentons ici a débuté en 2014 et a terminé en 2016. Nous avons commencé par une réunion dans laquelle on a posé des questions aux enseignants sur leur travail, y compris les difficultés auxquelles ils devraient faire face et nous avons filmé deux situations qu'ils ont choisies: (1) deux enseignants ont été filmés pendant qu'ils corrigeaient les évaluations; (2) deux autres enseignants ont été filmés dans deux cours de même niveau, pour aborder la difficulté à expliquer quelque chose aux apprenants quand eux-mêmes, enseignants, n'étaient pas sûrs de la réponse. Dans les deux cas, on a fait des autoconfrontations simples, croisées et des retours au collectif. Dans l'autoconfrontation simple que nous montrons dans cet article, issue de la situation (2), on voit comment cette méthode contribue pour que l'enseignante réfléchisse sur son travail et fasse des généralisations sur quelques manières de le réaliser.

Reprenons la thématique de cet entretien en autoconfrontation : les deux enseignants avaient été filmés de sorte à avoir du matériel de discussion sur leurs réactions quand les apprenants leur posent une question à laquelle ils ne savent pas répondre (situation 2$)^{2}$. Lors de l'entretien en autoconfrontation simple, une thématique liée à celle-là émerge : la difficulté à expliquer le vocabulaire en langue étrangère. Cette problématique apparaît à partir de la question de l'intervenante, ce qui montre le rôle de l'autre pour créer un autre point de vue. On voit s'installer un doute dans la manière de faire, qui n'est souvent pas interrogée par le sujet lui-même. A partir de ce doute, on voit apparaître un autre point de discussion : l' " excès » de vocabulaire dans les niveaux de base et la difficulté qu'ont les enseignants de le gérer. L'enseignante utilise, par la suite, un terme générique pour parler de ce vocabulaire : une avalanche de vocabulaire. L'usage de ce terme générique, qui n'est pas propre à la Didactique, montre une interprétation de la situation en tant qu'un problème " du monde », vu que les avalanches appartiennent à la sphère quotidienne, notamment celle des catastrophes naturelles. Même s'il ne s'agit pas d'un terme propre à la Didactique des Langues, retenons le fait qu'il s'agit d'un terme qui appartient au champ sémantique des « catastrophes » naturelles.

Lorsque l'enseignante reconstitue cet objet de discours (Mondada, 2002), pour le dire de plusieurs façons différentes, on voit apparaître une autre question : comment mémoriser ce vocabulaire, qui est le problème réel lié à l'enseignement-apprentissage d'une langue étrangère. Elle arrive ainsi à formuler une généralisation, qui la mène à co-construire, avec l'intervenant, un savoir sur l'apprentissage du vocabulaire : pour le mémoriser, une solution est demander une

\footnotetext{
${ }^{2}$ Dans les limites de cet article, nous avons choisi de présenter une analyse selon la construction des objets de discours, étant donné que d'autres types d'analyse ont déjà été publiés.
} 
production écrite pour qu'ils le réutilisent et qu'ils l'apprennent, le mémorisent. Voici l'extrait en question ${ }^{3}$ :

Extrait : Autoconfrontation simple avec V.

- 502P. ils posent beaucoup de questions non sur le vocabulaire?

- 503V. ouais ils posent mais :: tu dis :: dans ce cours-là ?.... au long du cours
$-504 \mathrm{P}$.
[oui au long du cours

- 505V. oui je crois que c'est normal hein qu'ils posent... normalement ils posent beaucoup de questions sur le vocabulaire... et je crois que je donne beaucoup d'espace aussi.. genre « ah ils parlent de crever :: là on commence à parler du pneu...on commence à parler de l'église... » et là quand je vais expliquer sur l'église je vais parler d'une autre chose qu'ils ne connaissent pas...donc :: on parle beaucoup de vocabulaire... je sais pas si c'est parce que c'est le niveau trois

- 506P. comment ça?

- $507 \mathrm{~V}$. ah parce qu'ils ont pas trop de vocabulaire encore donc $:$ : et le niveau trois... il y a beaucoup de vocabulaire c'est ça... les trois leçons... c'est de ::nourriture de ::vêtements... et des logements... et c'est la première fois qu'ils sont en train de voir ça donc c'est... une avalanche de :: de vocabulaire... c'est pas que moi je (amène) du vocabulaire c'est :: les unités qu'ils vont voir des choses qu'ils ont jamais vu donc $::$ dans une leçon :: ils vont apprendre ::les mots pour :: la nourriture après... la leçon/la leçon prochaine c'est des vêtements qui n'a rien à voir avec la nourriture... c'est tout un vocabulaire nouveau et :: long... c'est pas un vocabulaire deux ou trois mots... non... il y a plein de choses et :: d'ailleurs c'est un truc qu'ils se plaignent...plaint... dans la ::niveau trois qu'il y a trop de vocabulaire

- 508P. et quelle est ta perception de ça ? tu es d'accord avec eux ?

- 509V. oui... c'est une avalanche de vocabulaire... en même temps ::je pense que toutes les unités de base sont un peu une avalanche de vocabulaire parce qu'ils connaissent pas... je sais pas... j'ai pas donné de niveau deux niveau un pour savoir mais :: j'imagine que niveau un ça doit être une avalanche de vocabulaire aussi...s'ils connaissent rien...tout est nouveau... donc $::$ je sais pas si/si je suis d'accord avec ça mais :: je pense que c'est nécessaire du

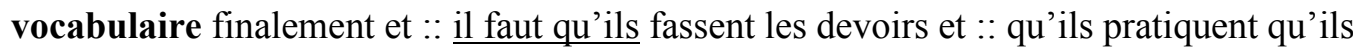
$\mathrm{met} / \mathrm{met} / \mathrm{mettent} \mathrm{en} \mathrm{pratique} \mathrm{parce} \mathrm{que} \mathrm{là} \mathrm{on} \mathrm{a} \mathrm{trois} \mathrm{heures} \mathrm{de} \mathrm{cours} \mathrm{par} \mathrm{semaine...} \mathrm{ils} \mathrm{vont} \mathrm{pas}$ retenir tout ce vocabulaire... donc :: c'est... il faut avoir... il faut travailler après aussi

- 510P. normalement tu fais quoi pour qu'ils travaillent ? pour qu'ils retiennent ça ?

- 511V. composition écrite... chaque cours ils me rendent une composition écrite

- 512P. tous les cours?

- $513 \mathrm{~V}$. oui ... tous les cours... je/quand/eh... j'arrive je rends le corrigé et là ils ()

- 514P. du coup j'imagine que tu fais toujours... tout le temps de corrections :: d'un cours à l'autre

- 515V. oui... d'un cours à l'autre oui

- 516P. et tu as combien de groupes?

- $517 \mathrm{~V}$. trois... mais je trouve que c'est essentiel hein ? une composition écrite... c'est pour ça que je fais ça... parce que le :: le cahier d'activités il est intéressant mais :: ils sont pas en train

\footnotetext{
${ }^{3}$ Dans l'extrait : V.: enseignante; P. : intervenante. Souligné : modalisations ; caractère gras : reprises autour de l'objet de discours « excès de vocabulaire »; caractère gras et italique : articulateurs logiques.
} 
de :: tu vois eh :: parfois ils ont compris la logique de l'exercice et c'est plus difficile quand $\underline{\text { il }}$ faut dire une chose par toi-même... et :: ils aiment bien... ils ont dit " ah c'est super ça...j'aime bien que... faire des compositions écrites... ça aide beaucoup $" . .$. je pense que c'est un cours par semaine donc $:: i$ ils ont le temps/assez de temps pour oublier... donc $::$ une composition écrite... par/par semaine c'est ça

\section{Source : Intervention (2014-2016) - Situation 2}

Dans l'extrait ci-dessus, on voit que l'idée de la grande quantité de vocabulaire, «beaucoup de vocabulaire », évolue pour «trop de vocabulaire» et se modifie jusqu'à se traduire par: une «avalanche de vocabulaire ». Attirons également l'attention sur le fait que l'articulateur logique « mais » apparaît 6 fois dans l'extrait précédent, ce qui montre la situation conflictuelle dans laquelle l'enseignante se retrouve, à partir du moment où l'intervenante lui pose une question sur le traitement du vocabulaire. Cependant, ce questionnement, loin de représenter un frein à l'action de l'enseignante, fonctionne ici comme un "moteur du développement », dans le sens de Clot $(1999,2001)$, car il permet que l'enseignante fasse une généralisation sur sa manière d'enseigner le vocabulaire, en la menant à comprendre les actions qui sont vraiment importantes dans cet enseignement : proposer des moments où les apprenants puissent réutiliser ce vocabulaire à l'écrit, afin qu'ils ne l'oublient pas et qu'ils le mémorisent.

Nous voyons ainsi le rôle de l'autoconfrontation pour faire réfléchir sur le travail enseignant, en proposant de revivre l'expérience vécue dans le cours comme un moyen de pouvoir vivre de nouvelles expériences, en « faisant autrement».

\section{CONCLUSIONS}

Dans cet article, nous avons discuté des rapports entre langage et travail en montrant comment les genres de texte peuvent être envisagés dans les situations de travail, soit pour comprendre le travail, lorsque le chercheur recueille les textes produits par les protagonistes dans leur contexte, soit pour transformer le travail, lorsque l'intervenant, le chercheur, le formateur, fait produire des genres, crée des verbalisations sur la situation de travail, dans le but de soutenir l'apprentissage, le développement, la formation.

Dans ce deuxième cas, nous voyons le potentiel formatif des genres comme lieu de verbalisations sur le travail. L'entretien en autoconfrontation apparait, dans ce cadre, comme une méthode qui promeut la réflexion sur le travail, le croisement des voix du langage intérieur reconstitué ou extériorisé, en montrant la transformation des sens attribués aux différentes manières de faire le travail et la coconstruction de savoirs sur le métier.

Nous ne l'avons pas montré dans cet article, mais, dans d'autres recherches (Lousada, 2013 ; Barricelli, Lousada, Bueno, 2017), nous avons constaté que d'autres genres sont également importants pour créer des verbalisations sur le travail, qui contribuent à la formation. Les récits et les rapports véhiculent l'influence des apprenants et les décisions futures pour le changement, de même que le rôle des prescriptions dans ce changement. Les journaux, très subjectifs par leur nature, montrent les sentiments sur les cours, sur soi-même et sur la profession. Les entretiens d'instruction au sosie, quant à eux, montrent une réflexion sur le travail, la projection des manières de faire le travail en permettant d'élargir les manières d'agir en situation de travail.

Dans tous ces exemples, on observe que les différents genres permettent les verbalisations sur le travail sous différentes conditions de production et d'organisation textuelle et des caractéristiques énonciatives, discursives et linguistiques spécifiques qui permettent de penser sur le travail et peuvent faire apparaître la voix du langage intérieur, dans le sens de 
Vygotski (1934/1997). Les entretiens en autoconfrontation ont montré le changement des sens attribués à quelques caractéristiques du travail enseignant, ainsi que la co-construction de nouveaux savoirs issus de la pratique quotidienne.

Dans cette perspective, le rôle de ces genres qui peuvent avoir des buts formatifs est essentiel pour changer les actions dans le monde du travail : il ne s'agit pas seulement d' « apprendre » le genre textuel, mais d'utiliser le langage, matérialisé dans des textes appartenant à des genres variés, pour transformer les situations de travail, changer les actions, les pratiques et les manières de faire le travail, enfin, modifier les représentations construites sur divers aspects du métier en créant possiblement de nouveaux savoirs. Les genres textuels à but formatif pourraient être vus, dans ce cadre, comme des transformateurs des pratiques d'enseignement et du travail enseignant.

\section{BIBLIOGRAPHIE}

Authier-Revuz, J. (2001). Palavras incertas: as não-coincidências do dizer. Campinas : Editora da Unicamp.

Barricelli, E., Lousada, E. G. \& Bueno, L. (2017). A instrução ao sósia : diálogos de confrontação sobre a atividade docente. In : M. A. A. Mascia, D. Anjos \& A. L. Smolka. (Eds.). Leituras de Vigotski (pp-109-130). Campinas : Mercado de Letras.

Boutet, J. (2001). La part langagière du travail : bilan et évolution. Langage et Société, 98(4), $17-42$.

Boutet, J. (2016). Atividades de linguagem em situação de trabalho. Parágrafo, 4(1), 91-97.

Bronckart, J.-P. (1999). Atividade de linguagem, textos e discursos: por um interacionismo sociodiscursivo. São Paulo : EDUC.

Bronckart, J.-P. (2004). Pourquoi et comment analyser l'agir verbal et non verbal en situation de travail. Agir et discours en situation de travail. Cahiers de la section des Sciences de l'Éducation, 103, pp. 11-131.

Bronckart, J.-P. (2008). O agir nos discursos: das concepções teóricas às concepções dos trabalhadores. Campinas : Mercado de Letras.

Bronckart, J.-P. (2019). Théories du langage. Bruxelles : Editions Mardaga.

Bulea, E. (2010). Linguagem e efeitos desenvolvimentais da interpretação da atividade. Campinas : Mercado de Letras. 
Bulea, E.; Bronckart, J-P. (2010). Les conditions d'exploitation de l'analyse des pratiques pour la formation des enseignants. LINGYARVM ARENA, 1, 43-60.

Clot, Y. (1999). La fonction psychologique du travail. Paris : PUF.

Clot, Y. (2001). Editorial. Clinique de l'activité et pouvoir d'agir, Education Permanente, 146, 7-16.

Clot, Y. (2008). Travail et pouvoir d'agir. Paris : PUF.

Clot, Y, Faïta, D., Fernandez, G. \& Scheller, L. (2001). Entretiens en autoconfrontation croisée : une méthode en clinique de l'activité. Education Permanente. Dossier : Clinique de l'activité et pouvoir d'agir. 146, 17-25.

Faïta, D. (2004). Gêneros de discurso, gêneros de atividade, análise da atividade do professor.

In: A. R. Machado (Ed.). O ensino como trabalho: uma abordagem discursiva. Londrina : EDUEL, pp. 55-80.

Faïta, D. \& Vieira, M. (2003). Réflexions méthodologiques sur l'auto-confrontation croisée. DELTA, 19(1), 123-154.

Friedrich, J. (2010). Lev Vygotski : médiation, apprentissage et développement. Une lecture philosophique et épistémologique. Genève : Carnets des Sciences de l'Education. FAPSE.

Koch, I. G. V. (2002/2013). Desvendando os segredos do texto. São Paulo : Cortez.

Kostulski, K. (2015). Dialogicité, dialogue et pensée dialogique du dialogue générique de l'audience correctionnelle au dialogue intérieur du procureur en situation de « plaider coupable ». Actes du 6ème séminaire international Vygotski, p. 361-380.

Laville, A. (2001). Repères pour une histoire de l'ergonomie francophone. Comptes rendus du congrès SELF-ACE. Les transformations du travail, enjeux pour l'ergonomie.

Lousada, E, G. (2006). Entre trabalho prescrito e realizado: um espaço para a emergência do trabalho real do professor. Thèse de Doctorat - Pontifícia Universidade Católica de São Paulo, São Paulo. 
Lousada, E. G. (2013). Textos na formação inicial de professores: o caso do relatório de estágio. In: L. Bueno, M. A. P. T. Lopes \& V. L. L. Cristovão. (Eds.). Gêneros textuais e formação inicial. (pp. 133-152). Campinas: Mercado de Letras.

Lousada, E. G. (2017). Le jeu de voix dans les verbalisations des enseignants débutants sur leur travail : prise de conscience, réflexion sur le métier et formation. In : Saussez, F. (Ed.). Travail et Apprentissages : Le formateur face à l'activité et son analyse : enjeux, écueils et perspectives. (pp. 103-120). Clamecy : Edition Raison et Passions.

Maingueneau, D. (2001). Análise de textos de comunicação. São Paulo: Contexto.

Mondada, L. (2002). Construction des objets de discours et catégorisation : une approche des processus de référentiation. Rev. de Letras, 24(1), 118-130. Recuperado de http://periodicos.ufc.br/revletras/article/view/20146

Nouroudine, A. (2002). A linguagem: dispositivo revelador da complexidade do trabalho. In: M. C. P. Souza-e-Silva \& D. Faïta. (Eds). Linguagem e trabalho: construção de objetos de análise no Brasil e na França. (pp. 17-30). São Paulo : Cortez.

Saujat, F. (2004). Comment les enseignants débutants entrent dans le métier », Formation et pratiques d'enseignement en questions. Revue des HEP de Suisse Romande et du Tessin, (1), 97-106.

Sitri, F. (1996). Interdiscours et construction de l'objet de discours. Linx, 8, 1-16. doi : 10.4000/linx.1158

Sitri, F. (2006). La construction des objets de discours à la lumière de la thématisation : les constituants thématiques multiples. Linx, 55, 115-129. doi : 10.4000/linx.396

Vygotski, L.S. (1934/1997). Pensée et langage. Paris : La Dispute.

Recebido em: 20 de março de 2020

Aceito em: 20 de julho de 2020

Publicado em Setembro de 2020 